\title{
Optimization of Dimensions of Cylindrical Piezoceramics as Radio-Clean Low Frequency Acoustic Sensors
}

\author{
M. Ardid, I. Felis, J. A. Martínez-Mora, and J. Otero \\ Institut d'Investigació per a la Gestió Integrada de les Zones Costaneres (IGIC), Universitat Politècnica de València (UPV), \\ 46730 Gandia, València, Spain
}

Correspondence should be addressed to M. Ardid; mardid@fis.upv.es

Received 19 April 2017; Revised 28 June 2017; Accepted 17 July 2017; Published 20 August 2017

Academic Editor: Carlos Ruiz

Copyright (C) 2017 M. Ardid et al. This is an open access article distributed under the Creative Commons Attribution License, which permits unrestricted use, distribution, and reproduction in any medium, provided the original work is properly cited.

Circular piezoelectric transducers with axial polarization are proposed as low frequency acoustic sensors for dark matter bubble chamber detectors. The axial vibration behaviour of the transducer is studied by three different methods: analytical models, FEM simulation, and experimental setup. To optimize disk geometry for this application, the dependence of the vibrational modes in function of the diameter-to-thickness ratio from 0.5 (a tall cylinder) to 20.0 (a thin disk) has been studied. Resonant and antiresonant frequencies for each of the lowest modes are determined and electromechanical coupling coefficients are calculated. From this analysis, due to the requirements of radiopurity and little volume, optimal diameter-to-thickness ratios for good transducer performance are discussed.

\section{Introduction}

The first discovery of the piezoelectric effect was found in 1880 by Jacques and Pierre Curie. They discovered that a quartz crystal submitted to mechanical action will produce an electrical potential that is proportional to the force applied. This effect is called direct piezoelectric effect. In the next year, Gabriel Lippmann described an analytical method for the reverse piezoelectric effect and the Curie brothers demonstrated this theory in the laboratory. Since its discovery, the piezoelectricity has been used in different fields such as defence, medical diagnosis, particle detection, ultrasonics, motors, and echolocation $[1,2]$. Due to the different vibrations modes in a piezoelectric material, the applications of this phenomenon can be different in each field. The first piezoelectric material used was the quartz; however, this material is not very good to transform the electrical energy into mechanic energy and its sensitivity is small. Later, in the middle of XIX century, lead zirconate titanate crystals (PZT) were developed. This material has a better sensitivity and frequency response. This material is produced using a very intense electrical field that polarizes the ceramic in a specific direction.
Piezoceramic transducers have usually a regular geometry and the knowledge of the behaviour of piezoceramic disks is important for transducer design and applications [3,4]. With these transducers, it is possible to generate vibrations from a few to several hundreds of $\mathrm{kHz}$, demonstrating its feasibility to be used as ultrasonic sensors and actuators in this frequency range.

For example, recent applications use piezoelectric sensors in order to detect the acoustic signal emitted by the interaction of elementary particles in a fluid target. In this sense, the dark matter detectors PICO bubble chambers use a superheated fluid target filled in a glass vessel $[5,6]$. Under some thermodynamic conditions, the particle interaction produces a bubble nucleation within the fluid and acoustic waves are emitted during the bubble growth. These sensors are glued to the external walls of the vessel that contains the metastable fluid. The acoustic discernment of the different acoustic events depends forcefully on the complete sensor properties and there are constraints as well in the little size and radiopurity of the piezoelectric ceramics [7]. These detectors use circular piezoceramics (both cylindrical and disk types) in the transducer design for a frequency bandwidth up to $150 \mathrm{kHz}$. These show the influence of bonding the ceramic 
to the vessel in its acoustic response. Among others, it is extracted that there is an increase of the sensors sensitivity, mainly in low frequencies. This is due to a better adaptation of acoustic impedances between the medium and the ceramic, through the glass of the vessel [7]. However, the first step in the design of the final transducer is the choice of the type and size of the ceramic taking into account its final use. For this, and since low radioactivity is a must, the amount of ceramic used in the transducer is a factor of great importance. This is because the ceramic material contains lead, which is usually accompanied with heavy radioisotopes that are alpha emitters and thus a source of background through alphaneutron reactions.

In this paper, we explain a proven methodology to study the optimization of this type of sensors. For this, several circular section (disks or cylinders) PIC255 piezoceramics with different width and height are studied with analytical and numerical methods and the results obtained are contrasted by experimental measurements. In previous works with circular PZT, some authors studied the natural vibration modes of axial symmetric piezoceramics using Finite Element Methods (FEM) [3] focusing on the efficiency and transducer design. Theoretical analysis contrasted with experimental measurements of resonant vibration with interferometry and laser Doppler vibrometer have been used [8]. Additional characterization methods and theoretical approach formulas can also be found. Here, the main parameters of piezoceramics will be compared: the resonance, $f_{r}$, and antiresonance, $f_{a}$, frequencies of the electrical impedance and its amplitude, the product of each frequency resonance and the length (thickness, $t$, or diameter, $d$ ) associated with the mode of vibration $\left(f_{r} \cdot d\right.$ and $\left.f_{a} \cdot t\right)$, and the piezoelectric coupling factor, $k^{2}=f_{a}^{2}-f_{r}^{2} / f_{a}^{2}$. Moreover, the ratio of coupling factors $\left(k_{1} / k_{2}\right)$ of the lowest piezoelectric modes gives us a quantified estimation of the energy distribution in this frequency range. The conclusions of these studies lead us to have design principles to select a specific piezoceramic circular geometry with a radio-clean piezoelectric material that can be used in the next generation of dark matter bubble chamber detectors (PICO 500 L) [9].

\section{Theoretical Analysis}

As discussed, the optimization of the volume is based on a free bounded circular ceramic such as that shown in Figure 1 that shows the geometrical scheme of a piezoceramic disk with thickness $t$ and radius $R$. The piezoelectric disk is polarized along the thickness ( $z$-axis), and the two opposite plane faces are covered with complete electrodes.

2.1. Analytical Method. The vibrational behaviour of a circular piece of the characteristics shown in Figure 1 can be obtained from the constitutive equations for a piezoelectric material. See [10-12] for a comprehensive review in this regard. For the purposes of this text, it is important to note that there are three different modes of vibration of thin piezoceramic disks: tangential, transverse, and radial extensional modes. However, theoretical and experimental

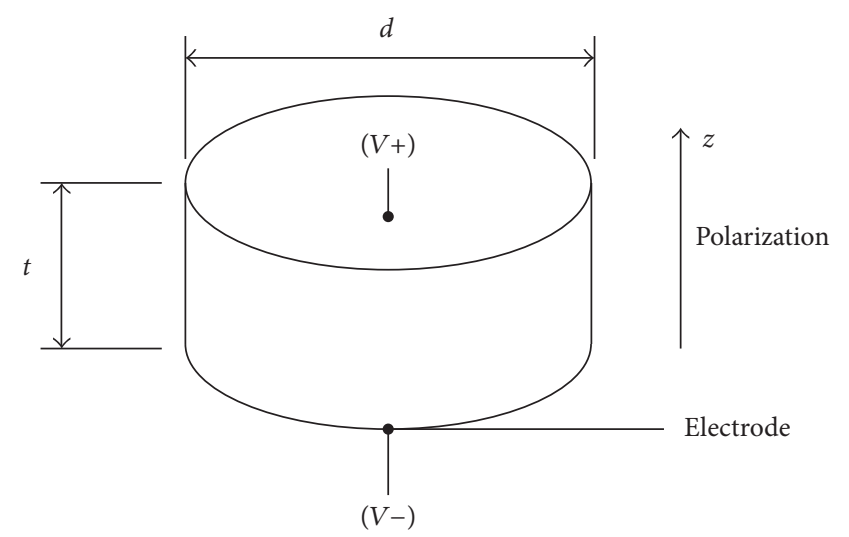

FIGURE 1: PZT transducer with longitudinal polarization.

analysis of these modes [8] shows that only the resonance frequencies of the radial vibrations modes can be measured in an impedance analysis. For this reason, we only analyse the vibrational characteristics of the extensional modes.

Assuming that there is a harmonic radial vibration in time $(t)$ with a known frequency $(\omega)$, the radial displacement in the median plane along the radius $r$ can be expressed as

$$
U_{r}(r, t)=U(r) e^{i \omega t} .
$$

The piezoelectric equations lead to the following general solution for the radial component of the vibration:

$$
U(r)=A J_{1}(\alpha r),
$$

where $J_{1}$ is the Bessel function of the first kind, for order 1 , and the parameters $A$ and $\alpha$ are given by

$$
\begin{aligned}
& A=\frac{2 V d_{31}\left(1+v_{p}\right)}{\left(1-v_{p}\right) J_{1}(\alpha R)-\alpha R J_{0}(\alpha R)} \frac{R}{t}, \\
& \alpha=\omega \sqrt{\rho s_{11}^{E}\left(1-v_{p}^{2}\right)}
\end{aligned}
$$

where $v_{p}=s_{12}^{E} / s_{11}^{E}$ is Poisson's ratio, $R$ is the radius, $\rho$ is the density of the material, $V$ is the electrical potential difference between the electrodes, and $s_{11}^{E}, s_{12}^{E}$, and $d_{31}$ are mechanical and piezoelectric coefficients that can be seen in the Appendix.

The resonance and antiresonance frequencies can be found where the current becomes infinite and becomes zero, respectively. With this, the characteristic equations for both resonance frequencies can be written as

$$
\begin{aligned}
& \eta J_{0}(\eta)=\left(1-v_{p}\right) J_{1}(\eta), \\
& \eta J_{0}(\eta)=\left[1-v_{p}+\left(1+v_{p}\right) \frac{k_{p}^{2}}{k_{p}^{2}-1}\right] J_{1}(\eta),
\end{aligned}
$$

where $J_{0}$ and $J_{1}$ are the Bessel function of the first kind, for orders 0 and 1 , respectively, and $\eta=\alpha R$. Equation (4) is the well-known result for two-dimensional analysis of the radial modes [8]. 
TABLE 1: Geometries modelled in FEM simulations.

\begin{tabular}{lcccccccccccccccccccc}
\hline$d$ & 10 & 10 & 10 & 10 & 10 & 10 & 5 & 10 & 7 & 10 & 13 & 15 & 18 & 20 & 22 & 24 & 25 & 30 & 35 & 40 \\
{$[\mathrm{~mm}]$} & & & & & & & & & & & & & & & & & & \\
$t$ & 20 & 17 & 15 & 10 & 7 & 5 & 2 & 3 & 2 & 2 & 2 & 2 & 2 & 2 & 2 & 2 & 2 & 2 & 2 & 2 \\
{$[\mathrm{~mm}]$} & 0.5 & 0.6 & 0.7 & 1 & 1.4 & 2 & 2.5 & 3.3 & 3.5 & 5 & 6.5 & 7.5 & 9 & 10 & 11 & 12 & 12.5 & 15 & 17.5 & 20 \\
$d / t$ & 0.5 &
\end{tabular}

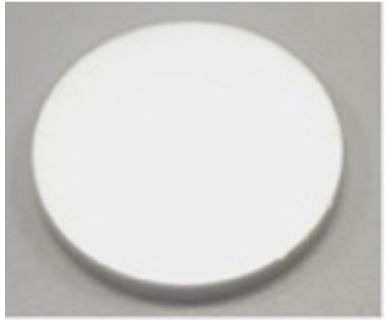

(a)
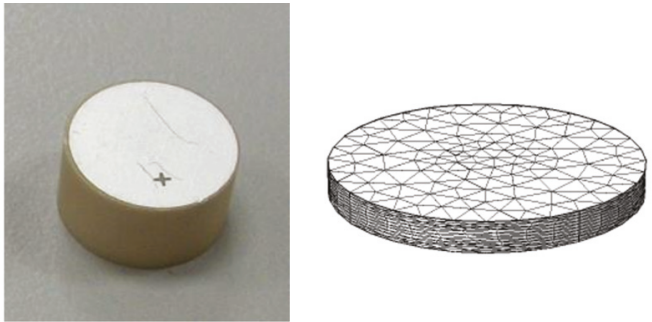

(b)

FIGURE 2: Modelling with FEM. (a) Photograph of a sample of circular piezoelectric transducer and (b) 3D meshing model used in numerical simulations.

From (4) and (5), the resulting resonant and antiresonant frequencies of the radial extensional mode for a circular piezoelectric piece with free boundary conditions can be expressed as

$$
f=\frac{\eta}{2 \pi R} \sqrt{\frac{1}{\rho s_{11}^{E}\left(1-v_{p}^{2}\right)}} .
$$

As mentioned, the basis for comparing these analytical results with experimental measurements or numerical simulations of the electrical impedance is that it reaches a local minimum when the sample vibrates at the resonance frequency and a maximum at antiresonance.

2.2. Numerical Simulation Method. To simulate the behaviour of piezoelectric ceramics, the COMSOL Multiphysics software has been used. With this, it has obtained the impedance curves that have been used in this study. In the sizing of tetrahedral meshing elements, it was taken into account that the smaller wavelength (maximum frequency) was discretized in almost ten parts. A mechanical free boundary condition was set in all the contours of the transducer and uniformly electrical open-circuit voltages were set to the electrodes. The samples were polarized in the length direction (Figure 1) and the applied voltage was set to $500 \mathrm{mV}$.

In Figure 2, an example of the type of mesh used to discretize the solutions is shown. The number of meshing elements with tetrahedral structure was 6698. Since the electrical impedance measurements were made in air, a surface boundary impedance of $450 \mathrm{kRayl}$ was used.

Table 1 shows the thickness $(t)$ and diameter $(d)$ of the different cylindrical ceramics that have been simulated in order to study different vibrational modes. The selected dimensions are the typical ones used in PICO detectors. In addition, it has been decided to use a larger set of ceramics of $t=2 \mathrm{~mm}$ to study the variation with the diameter and a set
TABLE 2: Piezoelectric ceramics evaluated.

\begin{tabular}{lcccc}
\hline$d$ & 10 & 10 & 20 & 25 \\
{$[\mathrm{~mm}]$} & 5 & 2 & 2 & 2 \\
$t$ & 2 & 5 & 10 & 12.5 \\
\hline $\mathrm{mm}]$ & 2 & & & \\
$d / t$ &
\end{tabular}

of ceramics with $d=10 \mathrm{~mm}$ to study the variation with the thickness.

The geometries with a $d / t$ ratio equal to or more than 5 can be considered as disks [3]. The smaller the $d / t$ ratio, the more the ceramic behaves as a cylinder. With this in mind, the FEM method can be used to study the ceramics with different radius and length in order to obtain the resulting impedance response and the frequencies and amplitudes of each peak. Subsequently, the resulting behaviour will be studied taking into account the volume of each testing piece.

\section{Experimental Setup}

In order to contrast theoretical predictions of models with experimental results, we have measured the bandwidth frequency electrical impedance response of several cylindrical PIC 255 piezoceramics with different diameter and thickness (see Table 2). The measurement was done through the resonance method by using a Wayner Kerr Electronics 6500P LF impedance analyser. The samples are held in air only by three metal wires at the bottom and another one at the top to avoid loads and constraints for the vibration. Figure 3 shows the experimental setup to measure a piezoceramic with $d=$ $25 \mathrm{~mm}$ and $t=2 \mathrm{~mm}$.

When the ceramic is excited in a resonance frequency of a radial vibration, the value of the impedance reaches a minimum and the admittance is maximum. 

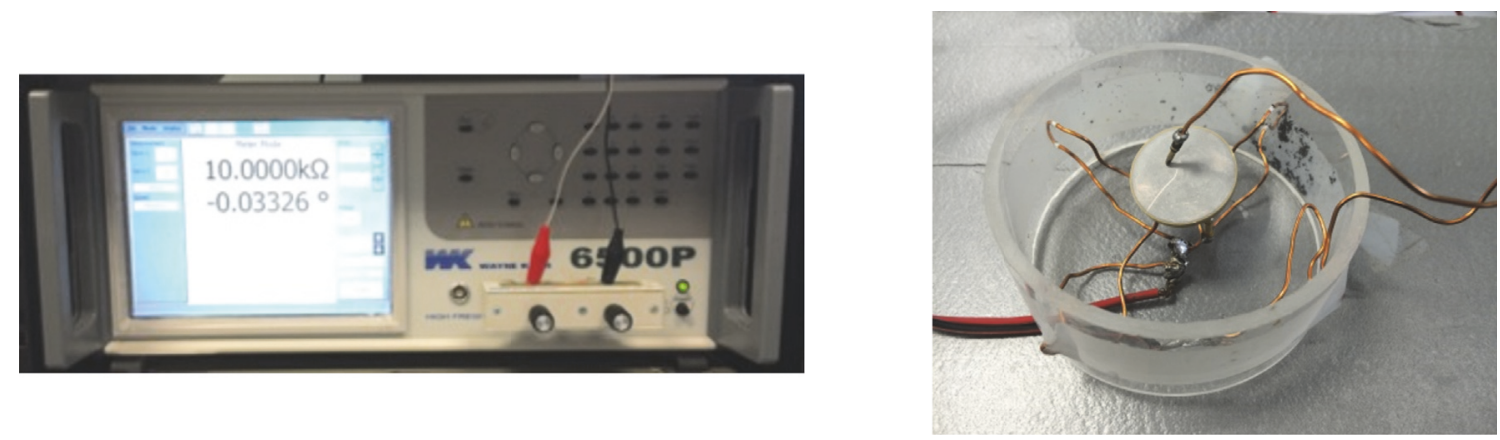

FIGURE 3: Experimental setup to measure the electrical impedance of the ceramics.

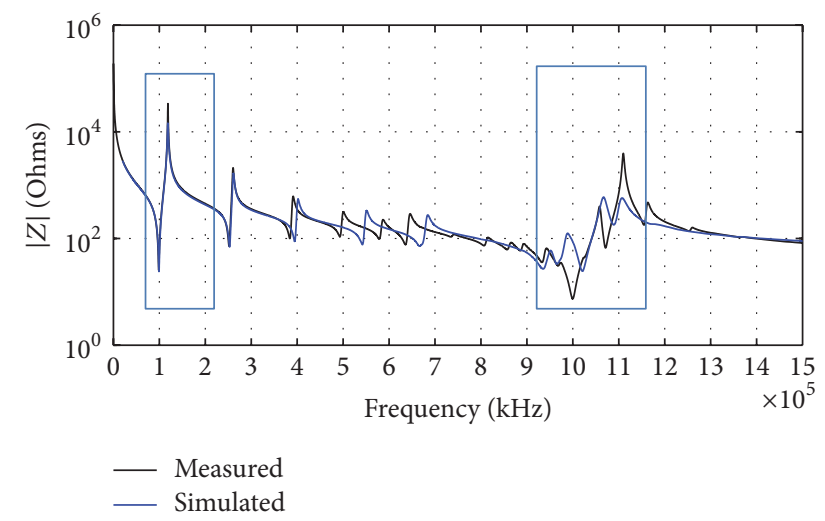

FIGURE 4: Comparison of impedance modulus obtained from FEM simulation and measurements.

\section{Results}

4.1. Width Frequency Band Impedance. Figure 4 shows both numerical and measured results for one of the studied transducers $(d=25 \mathrm{~mm}$ and $t=2 \mathrm{~mm})$. The frequency was swept from $100 \mathrm{~Hz}$ to $1.5 \mathrm{MHz}$ with an increment step of $100 \mathrm{~Hz}$. The modulus of the electrical impedance obtained is shown. We can distinguish between the low frequency radial modes and the high frequency thickness modes. The local minima and maxima appearing in the impedance curve correspond to resonance and antiresonance frequencies, respectively [13].

For the first three modes of Figure 4 that correspond to radial modes (left square), a good agreement, either in the frequency position of the peaks or in their amplitude, can be seen with a difference smaller than $0.01 \%$ in frequency and smaller than $0.05 \%$ in amplitude. For the thickness mode (right square), it should be pointed out that there is a large relative deformation and the effects of the losses are larger than those in radial modes. Anyway, we are not interested in very high frequencies, and thus the high frequency thickness modes are not of application in this work. Even so, when the $d / t$ ratio is smaller and smaller, this mode becomes of lower frequency, and experimental results and the simulations are in better agreement.

4.2. Modes Behaviour. With these experimental, numerical, and analytical methods, the relationship between the

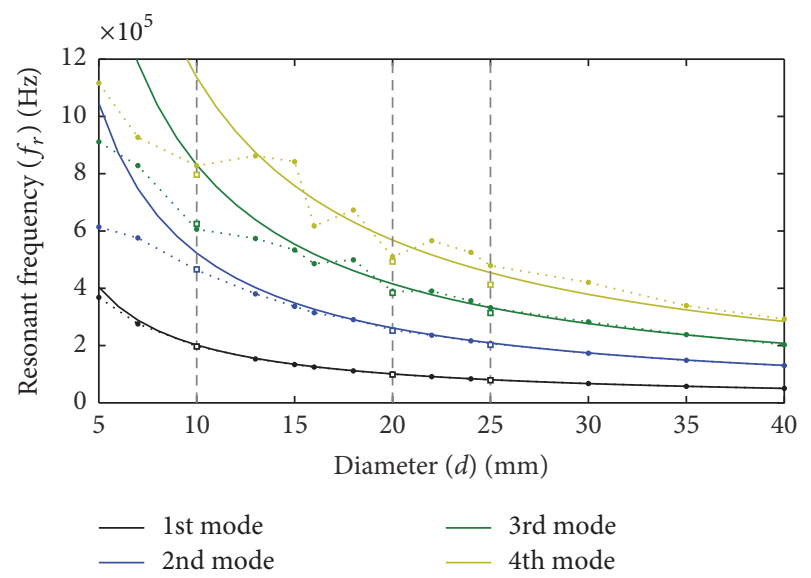

FIGURE 5: Dependence of the resonance frequencies with the diameter.

resonance frequencies and geometry parameters of the disk have to be studied. All these approaches will be taken into account and the results will be compared and discussed. For instance, Figure 5 shows the relationship between the frequency $f$ and diameter $d$ of the transducer for the first four modes when the thickness is $t=2 \mathrm{~mm}$. The solid line represents the analytical solutions, the circle-symbols represent the numerical simulation results, and the dashed line with squares denotes the situation of $d=25,20$, and $10 \mathrm{~mm}$ related to the experimental measurements.

Generally, the analytical and numerical results are in good agreement except for higher modes than the third one. In this range, the frequencies are close to the thickness vibration mode frequencies of the piezoelectric cylinder and strong coupling vibration of thickness and radial direction occurs. In addition, in these regions, the analytical solutions may have some errors due to the small diameter-to-thickness $(d / t)$ ratio; also the analytical method is based on a membrane theory [8]. We can see that the resonance frequency increases with decreasing diameter in a nonlinear relationship, especially for small diameters.

Figure 6 shows the relationship between the frequency $f$ and thickness of the transducer $t$ for the first four modes in the calculation for $d=10 \mathrm{~mm}$. The dashed line denotes the situation of $d=2$ and $5 \mathrm{~mm}$. Both experimental and simulated 


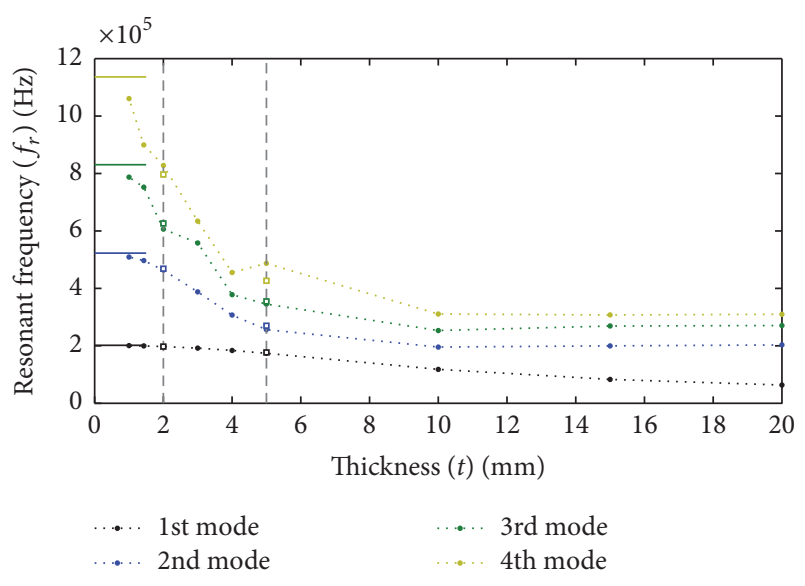

Figure 6: Resonant frequencies as a function of the thickness.

values show that frequency decreases with an increase in thickness and it converges to the analytical solution for low thickness. Since the analytical solution is valid only for ideal disks, it is independent of the thickness. For this, the analytical solutions are constant and close to the FEM solutions for larger diameter-to-thickness ratio, that is, for small thickness.

4.3. Frequency Constant. The frequency coefficient $N$ describes the relationship between the geometrical dimensions of a body and the corresponding (series) resonant frequency. When mechanical free piezoelectric ceramic element is excited with an alternating electric field and there is an impedance minimum, the radial resonance frequency matches with the series resonance frequency. The relationship between the radial mode resonance frequency constant, $N_{p}$, and the diameter of the ceramic element, $d$, is expressed by $N_{p}=f_{r} \cdot d$. At higher frequencies, there is another impedance minimum, the thickness resonance frequency. Thickness mode frequency constant, $N_{t}$, is related to the thickness of the ceramic element, $t$, by $N_{t}=f_{a} \cdot t$. Figure 7 shows the relation $f_{r} \cdot d$ to different $d / t$ ratio obtained both in FEM methods (dashed line) and experimentally (squares). It can be seen that when $d / t>10$ the frequency coefficient decreases significantly. This behaviour offers a geometrical distinction between disks and cylinders. In accordance with [1], the measures of resonant and antiresonant frequencies of a piezoelectric disk should be made better in terms of $d / t$ ratio than the conventional standards of measurement of piezoelectric coefficients $[14,15]$. The black solid line indicates the frequency constant $N_{p}$ in the theoretical model, which supports the above statement.

4.4. Thickness Mode Behaviour. Although high modes present a larger deviation between experimental and predicted results, it is interesting to show the distinction between antiresonant frequency constant in low and high modes. Figure 8 shows the value of $N_{t}=f_{a} \cdot t$ for the first four low modes (radial modes). As expected, for lower $d / t$ ratio, $N_{t}$ increases. Both numerical and analytical results agree

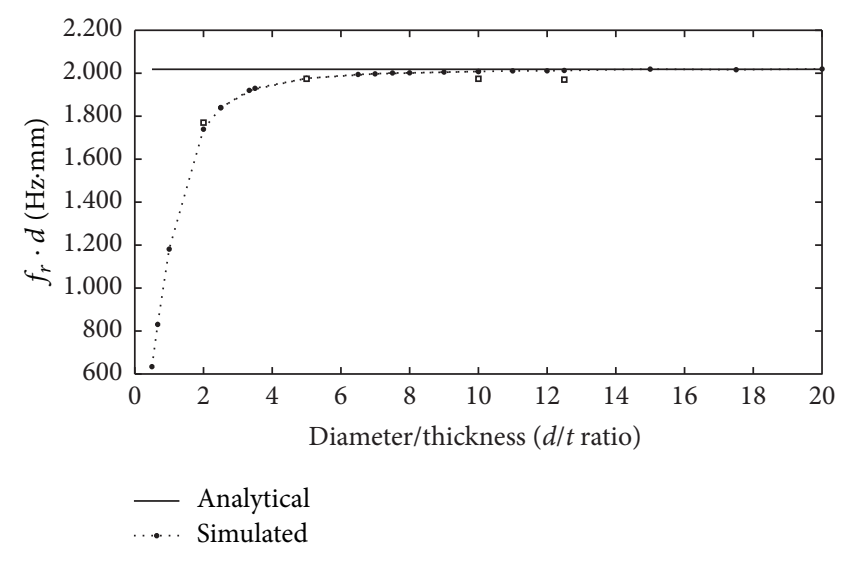

FIGURE 7: Frequency coefficient.

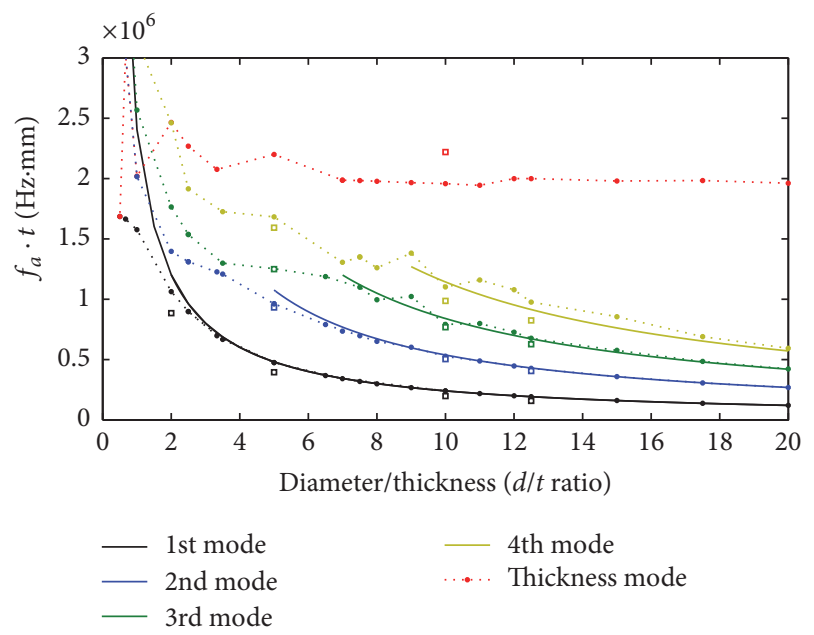

FIGURE 8: Frequency coefficient from antiresonant frequencies and thickness.

with experimental values above $N_{t}=1$. However, there is a clear trend upwards for lower $d / t$ ratios. Additionally, $N_{t}$ for thickness mode is shown. Since there is no thickness variation of these measures, the frequency constant remains quite constant and it is quite different to the low modes pattern. This behaviour shows clearly the difference between radial and thickness modes in piezoelectric disks. With this, we can predict that, for low $d / t$ ratios, both radial and thickness modes will be at similar frequencies, being possible that the thickness mode frequency is lower than the radial one.

4.5. Electromechanical Coupling Coefficients. The electromechanical coupling coefficient is an important characteristic of piezoelectric ceramics used to measure the energy conversion efficiency. The dynamic electromechanical coupling coefficient, proposed by Mason et al., can be obtained by measuring the resonant and antiresonant frequencies through the following expression $[16,17]$ :

$$
k=\sqrt{1-\left(\frac{f_{r}}{f_{a}}\right)^{2}} .
$$




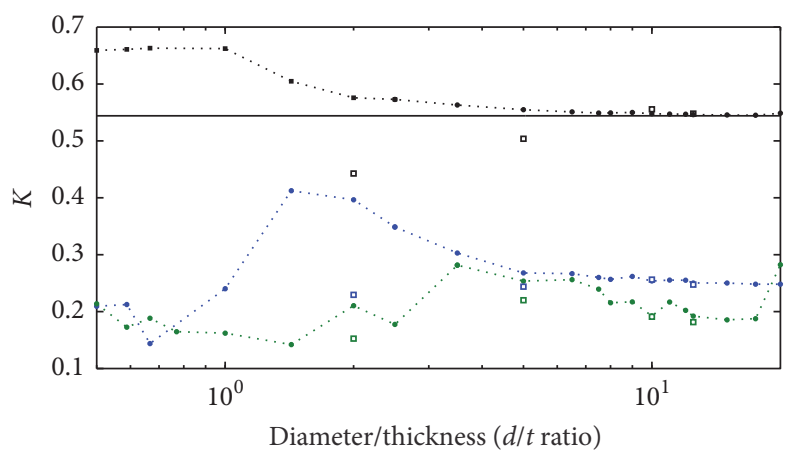

... 1st mode

.... 2nd mode

.... 3rd mode

Figure 9: Behaviour of the electromechanical coupling coefficients K obtained by theory, FEM simulations, and experimental measures for the lower extensional vibration modes.

This coefficient is of particular interest in designing piezoelectric elements. From Figure 9, we can find the value of $k$ for each of the extensional modes in function of the $d / t$ ratio.

As mentioned above, the theoretical approach used in this study is based on the assumption that the piezoelectric element is thin so that the theoretical predictions of resonant frequencies are accurate only if the diameter-to-thickness ratio $d / t$ is large. Figures 7 and 8 show the discrepancy between the analytical model and numerical simulations of the resonant frequencies for extensional vibrations in the performed values of $d / t$ ranging from 1 to 20 . It can be seen that there is a good agreement for $d / t>6$ with discrepancies within $2 \%$ for the first three extensional vibration modes. For the specific case of the first mode, the theoretical prediction is excellent, with discrepancy for $d / t=10$ of less than $0.5 \%$.

In general, it can be seen that the best efficiency for lowest radial mode occurs when $d / t<1$ but there is a minimum in the second radial mode that suggests that there is a weak interaction between radial modes [18]. For intermediate $d / t$ ratio, from 1 to 10 , a complicated vibrational spectrum appears, increasing the coupling of higher modes. In ratios $d / t>10$, it can be seen that the coupling factors of the lowest mode remain constants and those of higher modes decrease smoothly [19]. These conclusions about the coupling coefficient $k$ could be of great importance for the transducer design.

\section{Discussion and Conclusions}

The above studies have been done in order to develop tools to optimize the design of piezoelectric sensors for dark matter bubble chamber detectors. These studies started investigating the vibration of radial extensional modes in piezoelectric disks with mechanical free boundary by an analytical model, numerical simulations, and experimental measurements. Results with the diameter-to-thickness $d / t$ ratios, ranging from 0.1 (cylindrical shape) to 20 (disk shape), are presented. It is shown that the results agree fairly well for the four lower resonant frequencies.

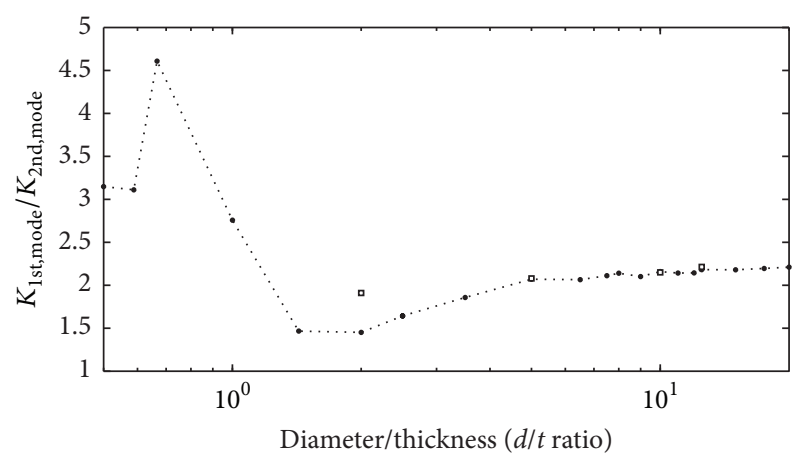

FIGURE 10: Ratio between the electromechanical coupling coefficients $k$ of the first and second mode.

In addition, electromechanical coupling $k$ indicates a different interaction between low and high modes for various $d / t$ values. The studies and results presented here are especially relevant for high frequencies, a complicated vibrational spectrum appears, and the transducer design becomes more complicated. The results show that a simple length expander mode, with minimum coupling to extraneous vibrational modes, can be achieved for $d / t<1$. These conclusions can be seen as well in Figure 10 where the ratio between the electromechanical coupling factor of the first mode $\left(k_{1 s t, m o d e}\right)$ and that of the second mode $\left(k_{2 \text { nd,mode }}\right)$ is shown.

From Figures 8 and 10, it is possible to understand the different behaviour according to $d / t$ ratio. In situations with $d / t<1$, the thickness mode frequency is below or quite close to the radial modes. Then, a high variation on the ratio between the electromechanical coupling coefficients $k$ can happen due to the different nature of the modes observing values of $k_{1} / k_{2}$ up to 4.5 , which results in a significant contrast in the efficiency between the two lower modes of the ceramic. For geometries $1<d / t<6$, the lowest resonance frequencies already correspond only to the radial mode, with relatively high values but with a lower coefficient $k_{1} / k_{2}$ (minimum is about 1.4), that is, with a greater interaction between modes, resulting in a more homogeneous response in this frequency range. For the case $d / t>6$, the resonance frequency decreases and the $k_{1} / k_{2}$ ratio increases but stabilizing at a value of approximately 2.2 .

In the design of piezoelectric ceramics for low background particle detectors, we must take into account the amount of material used in the construction of the sensors. In this sense, an additional parameter to take into account is the mass (or, equivalently, the volume) of the ceramic. Thus, since it depends on the square of the diameter and it is linear with respect to the thickness, we must reinterpret the previous results in terms of the volumetric ratio for each $d / t$ value.

In order to have a first approximation to this optimization problem, we compared the behaviour of three of the ceramics we have measured due to their different $d / t$ and volume values. Table 3 summarizes the results seen in this article.

Among these ceramics, for a wideband sensor application, no geometry meets the desired requirements of a small volume, low frequencies, and reduced $k_{1} / k_{2}$ ratio. However, considering that the electromechanical factor strongly 
TABLE 3

\begin{tabular}{lccccccccccccc}
\hline $\begin{array}{l}d \\
{[\mathrm{~mm}]}\end{array}$ & $\begin{array}{c}t \\
{[\mathrm{~mm}]}\end{array}$ & $\begin{array}{c}d / t \\
{[\mathrm{~mm}]}\end{array}$ & $\begin{array}{c}V \\
{\left[\mathrm{~mm}^{3}\right]}\end{array}$ & $\begin{array}{c}F_{r 1} \\
{[\mathrm{kHz}]}\end{array}$ & $\begin{array}{c}F_{a 1} \\
{[\mathrm{kHz}]}\end{array}$ & $\begin{array}{c}k_{1} \\
\text { exp. }\end{array}$ & $\begin{array}{c}k_{1} \\
\text { sim. }\end{array}$ & $\begin{array}{c}F_{r 2} \\
{[\mathrm{kHz}]}\end{array}$ & $\begin{array}{c}F_{a 2} \\
{[\mathrm{kHz}]}\end{array}$ & $\begin{array}{c}k_{2} \\
\text { exp. }\end{array}$ & $\begin{array}{c}k_{2} \\
\text { sim. }\end{array}$ & $\begin{array}{c}k_{1} / k_{2} \\
\text { exp. }\end{array}$ & $\begin{array}{c}k_{1} / k_{2} \\
\text { sim. }\end{array}$ \\
\hline 10 & 2 & 5 & 157.1 & 196.7 & 197.0 & 0.50 & 0.55 & 466.0 & 466.0 & 0.24 & 0.27 & 2.08 & 2.04 \\
10 & 5 & 2 & 392.7 & 177.0 & 177.0 & 0.44 & 0.57 & 270.0 & 270.0 & 0.23 & 0.40 & 1.91 & 1.43 \\
20 & 2 & 10 & 628.3 & 98.7 & 98.75 & 0.56 & 0.55 & 251.8 & 252.5 & 0.26 & 0.25 & 2.15 & 2.20 \\
\hline
\end{tabular}

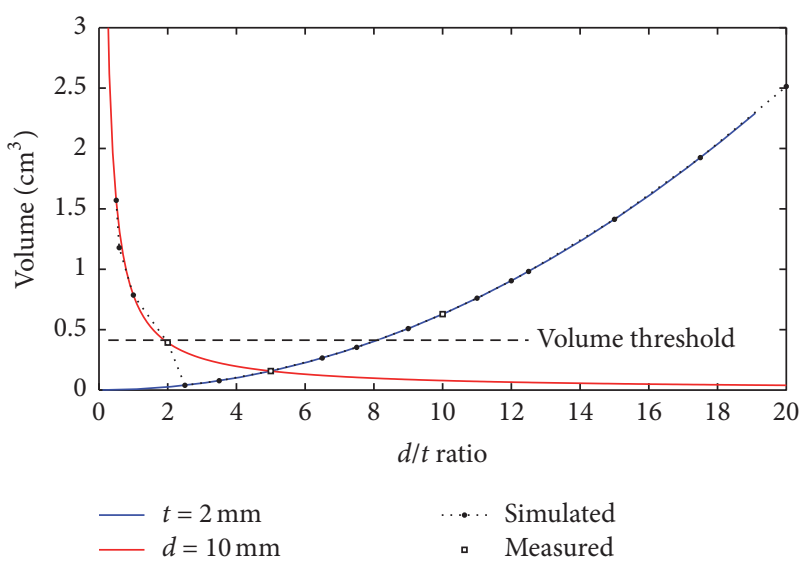

FIGURE 11: Relationship between volume and $d / t$ ratio for the studied geometries.

influences the acoustic behaviour of a piezoceramic used as acoustic receiver, we would choose the case $d / t=2$ because it presents a more flat acoustic response, with no prominent peaks [7].

Keeping this in mind, we can generalize the study to the set of simulated ceramics. Figure 11 shows the resulting volume of the studied geometries, distinguishing those corresponding to $d=10 \mathrm{~mm}$ (red line) and $t=2 \mathrm{~mm}$ (blue line). Hence, from radioactivity contamination conditions, we can establish a volume threshold. As an example, a threshold of $0.5 \mathrm{~cm}^{3}$ was chosen, so that, all ceramics below this threshold could be used matching the radio-clean limitation of the sensor.

Analogous to Figure 10, Figure 12 shows the ratio between electromechanical coupling factors of the lowest modes with respect to the volume of each sample. In this case, the curves of both geometries have been adjusted to the results by least squares, obtaining a good agreement with a relationship $k_{1} / k_{2} \sim-V^{-1}$. It is observed that, for ceramics with $t=2 \mathrm{~mm}$, we must use volumes smaller than $0.1 \mathrm{~cm}^{3}$ to have a coupling between the lowest modes, $k_{1} / k_{2}<2$. On the other hand, for geometries with $d=10 \mathrm{~mm}$ the situation is more relaxed and the ceramic measured is below the threshold of $0.5 \mathrm{~cm}^{3}$, matching therefore both criteria simultaneously.

Once the volumetric thresholds and coupling between modes have been established, it is necessary that the ceramic responds to the lowest possible frequency. We must be cautious because, in general, when the volume is smaller, so is the coupling, but the fundamental frequency increases. Figure 13 shows the fundamental resonance frequency for each studied sample, observing the geometry that minimizes the frequency response.

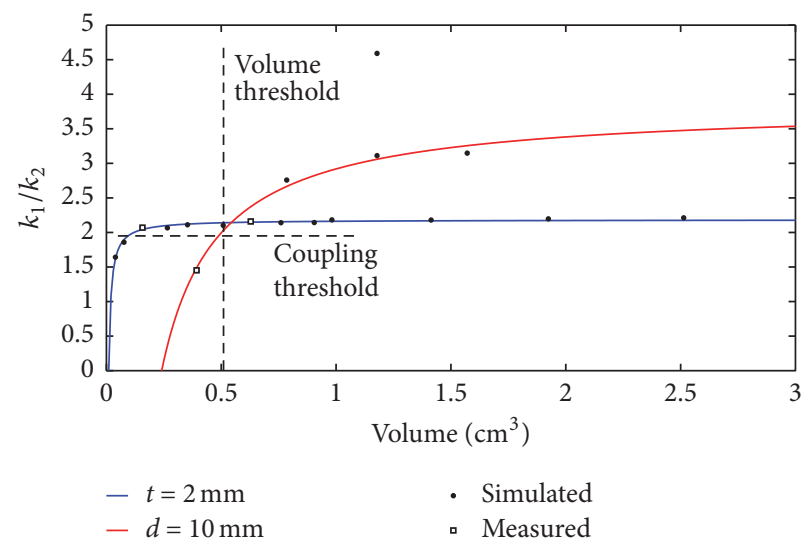

FIGURE 12: Ratio between the electromechanical coupling coefficients $k$ of the first and second lowest modes as a function of the volume.

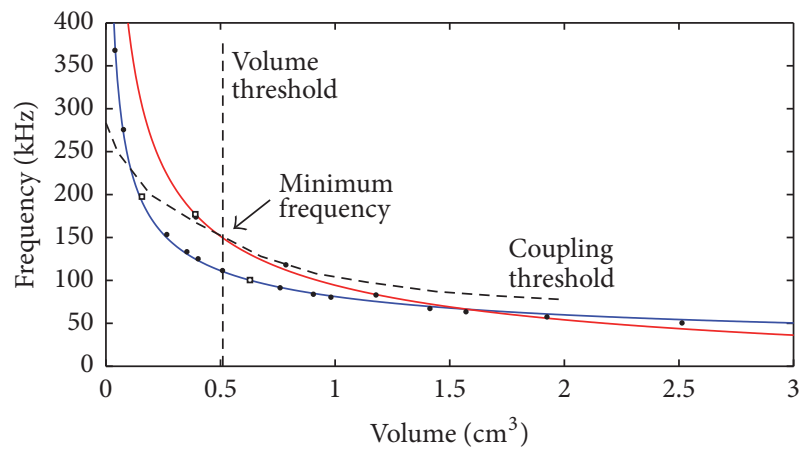

FIgURE 13: Fundamental frequency of the studied ceramics as a function of the volume.

From these three figures, we conclude that within $1.5<$ $d / t<9$ we can find geometries that are below the volumetric threshold (Figure 11). Among them, those that fulfil $1.5<$ $d / t<4.5$ satisfy a ratio $k_{1} / k_{2}<2$ (Figure 12), that is, a smaller coupling between lowest modes. Within the resulting geometries, the one with a lower fundamental frequency (Figure 13) is $d=10 \mathrm{~mm}$ and $t=7 \mathrm{~mm}$. We see that this relation is similar to the experimentally measured ceramic with $d=10 \mathrm{~mm}$ and $t=5 \mathrm{~mm}$, and therefore, it could also be a good option.

The compromise between low $k_{1} / k_{2}$ ratio and low fundamental frequency can also be studied from the measurement of the acoustic response (received voltage response) of each sample [7]. However, with the method presented in this text, we can limit the number of geometries that are adapted to the radio-clean requirements of the piezoelectric acoustic sensor 
design for the particle detectors such as the next generation of dark matter bubble chamber detectors.

\section{Appendix}

The properties of piezoceramics used in this study PIC255 are detailed:

$$
\begin{aligned}
& S^{E}=\left(\begin{array}{cccccc}
1.59 & -0.57 & -0.74 & 0 & 0 & 0 \\
-0.57 & 1.59 & -0.74 & 0 & 0 & 0 \\
-0.74 & -0.74 & -2.10 & 0 & 0 & 0 \\
0 & 0 & 0 & 4.50 & 0 & 0 \\
0 & 0 & 0 & 0 & 4.50 & 0 \\
0 & 0 & 0 & 0 & 0 & 4.32
\end{array}\right) \\
& \cdot 10^{-11}[1 / \mathrm{Pa}] \text {, } \\
& d=\left(\begin{array}{cccccc}
0 & 0 & 0 & 0 & 535 & 0 \\
0 & 0 & 0 & 535 & 0 & 0 \\
-174 & -174 & 394 & 0 & 0 & 0
\end{array}\right) \\
& \cdot 10^{-12}[\mathrm{C} / \mathrm{N}] \text {, } \\
& \varepsilon^{T}=\left(\begin{array}{ccc}
1649 & 0 & 0 \\
0 & 1649 & 0 \\
0 & 0 & 1750
\end{array}\right) \text {. }
\end{aligned}
$$

\section{Conflicts of Interest}

The authors declare that there are no conflicts of interest regarding the publication of this paper.

\section{Acknowledgments}

The authors acknowledge the financial support of the Spanish Ministerio de Economía y Competitividad (MINECO), Grants FPA-2015-65150-C3-2P, and Consolider MultiDark CSD2009-00064. Thanks as well are due to the PICO Collaboration members for the fruitful discussions, suggestions, and comments.

\section{References}

[1] A. A. Vives, "Preface," Piezoelectric Transducers and Applications, pp. VII-X, 2008.

[2] W. KochaNski, M. Boeff, Z. Hashemiyan, W. J. Staszewski, and P. K. Verma, "Modelling and numerical simulations of in-air reverberation images for fault detection in medical ultrasonic transducers: a feasibility study," Journal of Sensors, vol. 2015, Article ID 796439, 14 pages, 2015.

[3] Piezoelectric Ceramics: Principles and Applications, APC International, Ltd, 2nd edition, 2011.

[4] M. Martinez and A. Artemev, "A novel approach to a piezoelectric sensing element," Journal of Sensors, vol. 2010, Article ID 816068, 2010.
[5] C. Amole et al., "Dark matter search results from the PICO-60 $\mathrm{CF}_{3}$ I bubble chamber," Physical Review D, vol. 93, Article ID 052014, p. 05, 2016.

[6] C. Amole, "Dark Matter Search Results from the PICO-2L C $\mathrm{C}_{3} \mathrm{~F}_{8}$ Bubble Chamber," Phys. Rev. Lett, vol. 114, Article ID 231302, 2015.

[7] I. Felis, J. A. Martínez-Mora, and M. Ardid, "Acoustic sensor design for dark matter bubble chamber detectors," Sensors (Switzerland), vol. 16, no. 6, article no. 860, 2016.

[8] C.-H. Huang, Y.-C. Lin, and C.-C. Ma, “Theoretical Analysis and Experimental Measurement for Resonant Vibration of Piezoceramic Circular Plates," IEEE Transactions on Ultrasonics, Ferroelectrics, and Frequency Control, vol. 51, no. 1, pp. 12-24, 2004.

[9] M. Bou-Cabo, I. Felis, and M. Ardid, "Acoustic studies for alpha background rejection in dark matter bubble chamber detectors," in Proceedings of the 4th International Workshop in Low Radioactivity Techniques 2013, LRT 2013, pp. 142-147, Assergi, Italy, April 2013.

[10] D. Sun, S. Wang, S. Hata, and A. Shimokohbe, "Axial vibration characteristics of a cylindrical, radially polarized piezoelectric transducer with different electrode patterns," Ultrasonics, vol. 50, no. 3, pp. 403-410, 2010.

[11] H. A. Kunkel, S. Locke, and B. Pikeroen, "Finite-Element Analysis of Vibrational Modes in Piezoelectric Ceramic Disks," IEEE Transactions on Ultrasonics, Ferroelectrics, and Frequency Control, vol. 37, no. 4, pp. 316-328, 1990.

[12] N. N. Rogacheva, The Theory on Piezoelectric Shells and Plates, CRC Press, Boca Raton, Fla, USA, 1994.

[13] K. Kanazawa, S. M. Yoon, and N. J. Cho, "Analyzing spurdistorted impedance spectra for the QCM," Journal of Sensors, vol. 2009, Article ID 259746, 2009.

[14] T. L. Jordan and Z. Ounaies, "Piezoelectric Ceramic Characterization," Tech. Rep. NASA/CR-2001-21125, ICASE Report N0. 2001-28.

[15] “IEEE Standard on Piezoelectricity," Tech. Rep. ANSI/IEEE Std 176-1987.

[16] W. P. Mason, Piezoelectric Crystal and Their Application to Ultrasonics, Van Nostrand, New York, NY, USA, 1950.

[17] Q. Chen and Q.-M. Wang, "The effective electromechanical coupling coefficient of piezoelectric thin-film resonators," Applied Physics Letters, vol. 86, no. 2, Article ID 022904, 2005.

[18] J. Kocbach, Finite Element Modelling of Ultrasonic Piezoelectric Transducers, Department of Physics, University of Bergen, Bergen, Norway, 2000.

[19] A. Sáenz de Inestrillas, F. Inestrillas, M. Bou Cabo, J. M. Barreiro, and A. Reig, "Design and performance of a metalshielded piezoelectric sensor," Sensors, vol. 17, article 1284, 2017. 


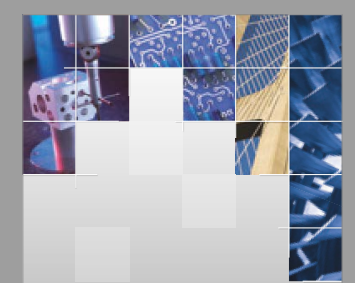

\section{Enfincering}
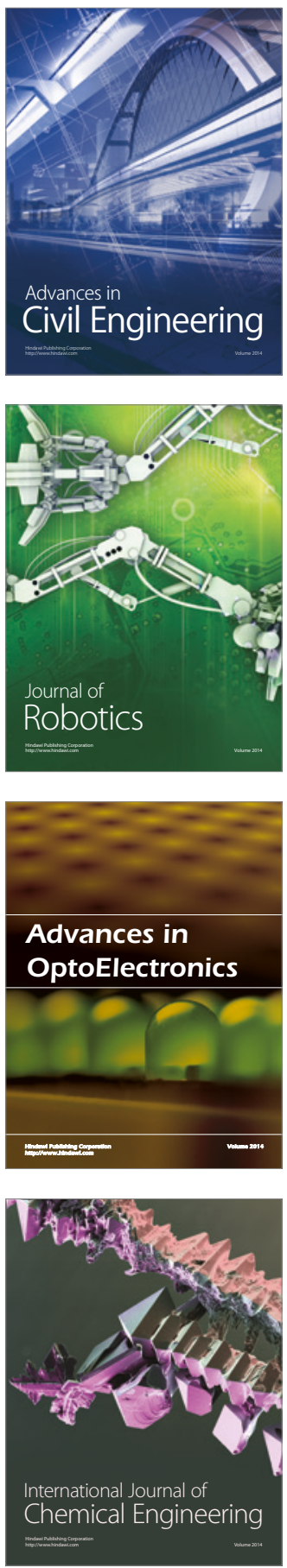

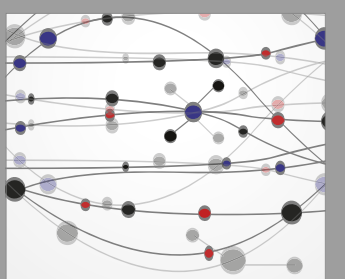

The Scientific World Journal

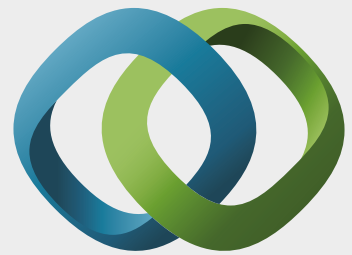

\section{Hindawi}

Submit your manuscripts at

https://www.hindawi.com
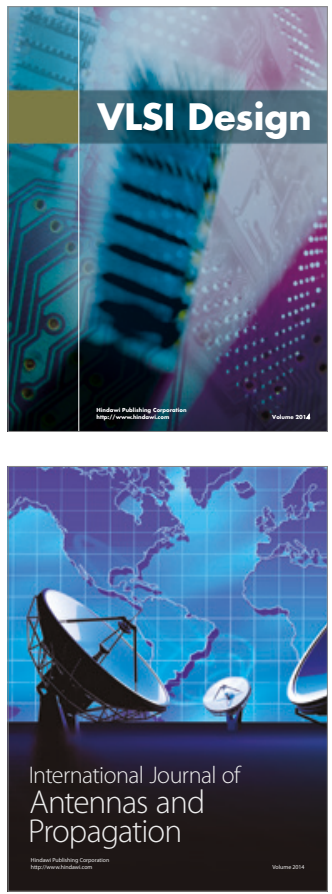

\section{Rotating}

Machinery
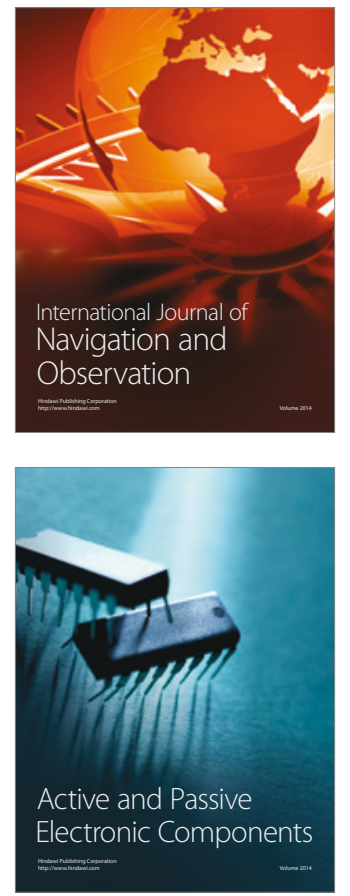
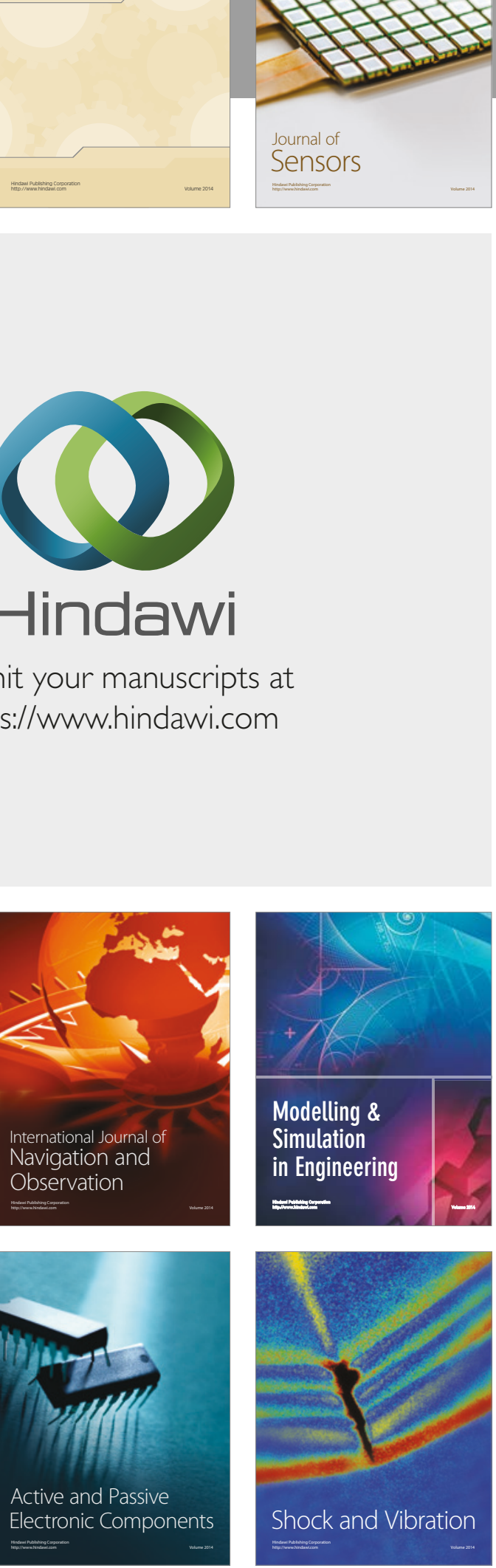
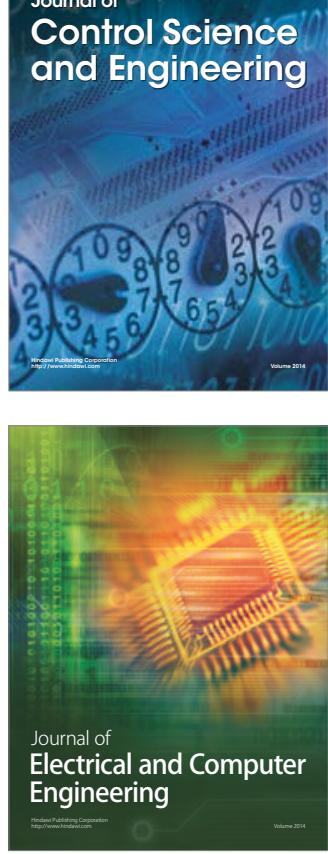

Distributed

Journal of

Control Science

and Engineering
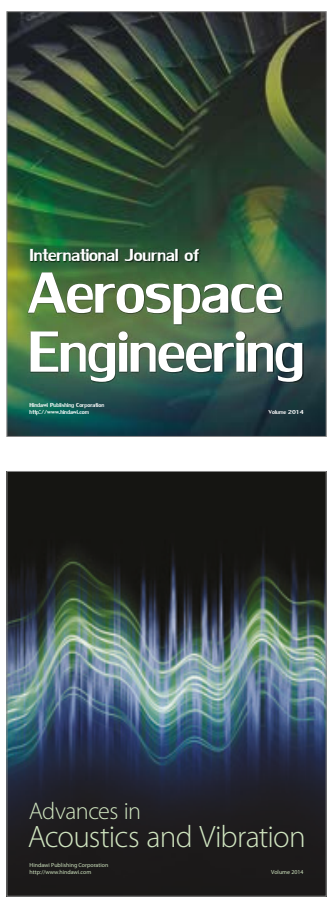

Sensor Networks 